\title{
O GERENCIAMENTO DE RISCOS EM PROJETOS GERENCIADOS POR ABORDAGENS ÁGEIS: UMA REVISÃO SISTEMÁTICA DA LITERATURA
}

\author{
RISK MANAGEMENT IN PROJECTS MANAGED BY AGILE APPROACHES: \\ A SYSTEMATIC REVIEW OF THE LITERATURE
}

\author{
(D) Pedro Jose Martins Alvarez Fernandes \\ Mestre em Administração \\ Universidade Nove de Julho - UNINOVE. \\ São Paulo, SP - Brasil. \\ pedromartins86@gmail.com \\ Roque Rabechini Jr. \\ Pós-doutor em Administração \\ Faculdade de Economia, Administração e Contabilidade da Universidade de São Paulo - FEA/USP. \\ São Paulo, SP - Brasil. \\ rabechinijr@gmail.com
} Resumo

Ainda que a gestão de riscos influencie os resultados dos projetos, sabe-se que há casos de fracasso cuja principal causa é a incorreta utilização das práticas dessa área de conhecimento. Sabe-se também que a abordagem ágil de gerenciamento de projetos e sua influência nos resultados dos projetos tem sido amplamente pesquisada, despertando interesse dos mais diversos no âmbito dos projetos. No entanto, a relação entre essas áreas é um fenômeno que não está consolidado na literatura especializada em gestão de projetos. O objetivo deste estudo foi verificar a evolução da relação entre a gestão de riscos e abordagem ágil. Espera-se, com isso, conhecer os principais riscos e as características dos modelos de gerenciamento de riscos em projetos gerenciados por abordagens ágeis. Este artigo apresenta uma análise exploratória realizada com base em um estudo bibliométrico seguido de uma revisão sistemática da literatura. Assim, verificou-se que a maioria dos 1023 artigos avaliados quantitativamente por meio da análise bibliométrica, está em sua fase inicial, pois foi somente publicada em conferências e congressos e, os 16 artigos avaliados qualitativamente na revisão sistemática de literatura tinham como principal objetivo, propor um modelo para o gerenciamento de riscos em projetos gerenciados por abordagens ágeis. Por fim, muitos riscos identificados na revisão sistemática estão associados ao fator pessoas, este que é um dos valores centrais da abordagem ágil, contrapondo os benefícios e as vantagens reconhecidos na literatura para esta abordagem.

Palavras-chaves: Gerenciamento de riscos. Ágil. Sucesso de projetos. Bibliométrico. Revisão sistemática da literatura.

\begin{abstract}
Although risk management influences the project results, it is known that there are cases of failure which main cause is the incorrect use of practices related to this knowledge area. It is also known that the agile approach for project management and its influence on project results has been widely studied, arousing the interests related to project management. Nevertheless, the relationship between these areas is a phenomenon that is not consolidated in the literature specialized in project management. The objective of this study was to verify the evolution of the relationship between risk management and agile approach, in order to identify main risks and the characteristics of risk management models in projects managed by agile approaches. This article presents an exploratory analysis based on a bibliometric study followed by a systematic review of the literature. Thus, it was found that most of the 1023 articles evaluated quantitatively through bibliometric analysis, were in their initial phase, as they were only published in conferences and congresses, and the 16 articles evaluated qualitatively in the systematic literature review
\end{abstract}


Fernandes, P. J. A., \& Rabechini, R. Jr. (2021, Ed. Esp. jan./abr.). O gerenciamento de riscos em projetos gerenciados por abordagens ágeis: uma revisão sistemática da literatura

had as their main objective, propose a model for risk management in projects managed by agile approaches.

Finally, many risks identified in the systematic review were associated with the people factor, which is one of the central values of the agile approach, contrasting the benefits and advantages recognized in the literature for this approach.

Keywords: Risk management. Agile. Project success. Bibliometric analysis. Systematic review of literature.

\section{Cite como - American Psychological Association (APA)}

Fernandes, P. J. A., \& Rabechini, R. Jr. (2021, Ed. Esp. jan./abr.). O gerenciamento de riscos em projetos gerenciados por abordagens ágeis: uma revisão sistemática da literatura. Revista de Gestao e Projetos (GeP), 12(1), 172-194. https://doi.org/10.5585/gep.v12i1.17817.

\section{Introdução}

Os projetos estão cada vez mais ganhando popularidade, tanto no setor público quanto no setor privado (Serrador \& Pinto, 2015). Entretanto, estudos comprovam que a taxa de sucesso destes ainda não é considerada constante, devido aos altos índices de atrasos, correções, abandonos e rejeições de projetos, mesmo que já implementados (Chow \& Cao, 2008). Com o intuito de reduzir a taxa de insucesso dos projetos, foram criadas as abordagens de gerenciamento de projetos, inicialmente as denominadas tradicionais, com processos mais rígidos e lineares e, posteriormente, as abordagens ágeis, essas que são mais flexíveis e adaptativas (Boehm, 2000, 2002).

$\mathrm{O}$ interesse pelas abordagens ágeis, conforme afirmam Senapathi e Drury-Grogan (2017) e Dingsøyr et al. (2018), tem sido cada vez maior no âmbito de projetos, relacionados ou não à tecnologia. Na visão de Serrador e Pinto (2015), este assunto desperta interesse aos pesquisadores de gestão de projetos uma vez que os projetos gerenciados por meio da abordagem ágil possuem índices de sucesso maior, quando comparados aos projetos gerenciados por meio da abordagem tradicional. No entanto, a abordagem tradicional conta com diversas propostas que são ainda incipientes na abordagem ágil, tal como o gerenciamento de riscos, esse que reduz as incertezas e aumenta as chances de sucesso dos projetos (Tavares et al., 2019). Corroborando com essa afirmação, diversos autores enfatizam a inexistência de processos específicos de gerenciamento de riscos em projetos gerenciados por abordagens ágeis (Alharbi \& Qureshi, 2014; Bumbary, 2016; Mousaei \& Javdani, 2018; Tomanek \& Juricek, 2015).

O impacto do gerenciamento de riscos no sucesso dos projetos ganhou relevância em estudos realizados na última década, enfatizando a importância da implementação de ferramentas, técnicas e processos relacionados ao gerenciamento de riscos nas organizações (Bakker et al., 2010; Carvalho \& Rabechini Jr., 2014). Por sua vez, Tavares et al. (2019) e Hammad et al. (2019) destacam ainda que as 
Fernandes, P. J. A., \& Rabechini, R. Jr. (2021, Ed. Esp. jan./abr.). O gerenciamento de riscos em projetos gerenciados por abordagens ágeis: uma revisão sistemática da literatura

falhas de projetos ágeis podem ser associadas à uma ineficaz gestão dos riscos desses projetos.

Considerando a lacuna mencionada, envolvendo o incipiente gerenciamento de riscos em projetos gerenciados por abordagens ágeis, o objetivo deste artigo foi verificar a evolução da relação entre esses dois constructos, assim como mapear os principais riscos e as características dos modelos existentes de gerenciamento de riscos para tais projetos, por meio de uma análise exploratória realizada com base em um estudo bibliométrico seguido de uma revisão sistemática da literatura para delineamento dos temas: gerenciamento de riscos e abordagem ágil de gerenciamento de projetos. O estudo bibliométrico foi composto por uma análise de citação, com o objetivo de medir a frequência com que as referências são citadas, seguida de uma análise de cocitação, identificando pares de artigos citados simultaneamente. A revisão sistemática da literatura teve como principal objetivo de compilar os riscos relacionados à abordagem ágil e as características dos modelos de gerenciamento de riscos existentes na literatura para projetos gerenciados por abordagens ágeis.

A contribuição teórica deste estudo é quanto à exploração do tema gerenciamento de riscos e sua relação com projetos gerenciados por abordagens ágeis, aproximando essas áreas.
As contribuições práticas estão em demonstrar a importância do gerenciamento de riscos para o sucesso dos projetos gerenciados por abordagens ágeis, assim como identificar os principais riscos e modelos de gerenciamento de riscos utilizados em tais projetos.

\subsection{Gerenciamento de riscos}

Risco é um evento que, se ocorrer, pode impactar de maneira positiva ou negativa o sucesso e os objetivos dos projetos (PMI, 2017). Por sua vez, o gerenciamento de risco é definido como um processo para identificação, avaliação e tratamento dos riscos, reduzindo-os a um nível aceitável pelas organizações (Stoneburner et al., 2002). Destaca-se que os projetos iniciam com um nível alto de exposição ao risco, diminuindo à medida que o projeto avança no tempo, pois o aumento das informações faz com que as incertezas diminuam (PMI, 2017).

Com o objetivo de facilitar a organização dos processos gerenciais e consequentemente a implementação de um adequado gerenciamento de riscos, as empresas passaram a utilizar os modelos de referência em gerenciamento de riscos disponibilizados na literatura relacionada a gerenciamento de projetos (Gusmão \& Moura, 2003), também apresentados resumidamente na Tabela 1 . 
Fernandes, P. J. A., \& Rabechini, R. Jr. (2021, Ed. Esp. jan./abr.). O gerenciamento de riscos em projetos gerenciados por abordagens ágeis: uma revisão sistemática da literatura

Tabela 1 - Principais modelos de referência em gerenciamento de riscos

\begin{tabular}{|c|c|c|c|c|}
\hline $\mathbf{N}^{\mathbf{o}}$ & Nome & Proprietário & Descrição & $\begin{array}{l}\text { Data de } \\
\text { Criação }\end{array}$ \\
\hline 1 & $\begin{array}{l}\text { Software Risk } \\
\text { Management }\end{array}$ & Barry Boehm & $\begin{array}{l}\text { Padrões focados para o desenvolvimento } \\
\text { de software. }\end{array}$ & 1989 \\
\hline 2 & CMMI (RSKM) & $\begin{array}{l}\text { Software Engineering } \\
\text { Institute - SEI }\end{array}$ & Específico para projetos de software. & 2002 \\
\hline 3 & ISO 31000 & $\begin{array}{l}\text { International Organization } \\
\text { for Standardization - ISO }\end{array}$ & $\begin{array}{l}\text { Utilização genérica, pois, atende qualquer } \\
\text { tipo de risco e segmento da indústria. }\end{array}$ & 2009 \\
\hline 4 & $\begin{array}{l}\text { Practice Standard } \\
\text { for Project Risk } \\
\text { Management }\end{array}$ & $\begin{array}{l}\text { Project Management } \\
\text { Institute - PMI }\end{array}$ & $\begin{array}{l}\text { Direcionado para o gerenciamento de } \\
\text { riscos de projetos. }\end{array}$ & 2009 \\
\hline
\end{tabular}

Fonte: Elaborado pelos autores.

Apesar de suas particularidades, os modelos de gerenciamento de riscos apresentado na Tabela 1 seguem, em linhas gerais, os passos definidos por Boehm (1991, 1989): (1) identificação de riscos, (2) análise de riscos, (3) priorização de riscos, (4) planejamento do gerenciamento de riscos, (5) resolução de riscos e (6) monitoramento de riscos.

Entretanto, Mousaei e Javdani (2018) enfatizam a inexistência de um modelo padrão de gerenciamento de riscos em projetos gerenciados por abordagens ágeis. Assim, citam que os pesquisadores geralmente baseiam seus estudos em modelos já consolidados na literatura, tal como o modelo proposto por Boehm (1989), este que não foi desenvolvido especificamente para projetos gerenciados por abordagem ágil.

\subsection{Abordagem ágil de gerenciamento de projetos}

A abordagem ágil abrange um conjunto de modelos, métodos e técnicas com o objetivo de lidar com o risco inerente a ambientes dinâmicos, por meio da flexibilidade, atividades interativas e entregas constantes, tanto para atividades de desenvolvimento de software quanto para atividades relacionadas ao gerenciamento de projetos (Boehm \& Turner, 2003; Highsmith \& Cockburn, 2001). Assim, tal abordagem consolidou-se como uma resposta aos problemas enfrentados pelas abordagens tradicionais, como o excesso de burocracia e inflexibilidade frente às mudanças de escopo, por meio da eliminação de barreiras e interação dos stakeholders (Boehm \& Turner, 2003).

Dybå e Dingsøyr (2008) comentam que a abordagem ágil ganhou popularidade após a elaboração do 'Manifesto Ágil', este que foi descrito por gestores de projetos experientes em meados de 2001. Destacam ainda a existência de diversos estudos comparando as características e eficácia das abordagens tradicionais e ágeis. Portanto, a Tabela 2 sumariza as principais diferenças entre essas abordagens, revelando que a abordagem tradicional se concentra nos processos e requisições detalhadas previamente, enquanto as abordagens ágeis fomentam o dinamismo e a comunicação das pessoas. 
Fernandes, P. J. A., \& Rabechini, R. Jr. (2021, Ed. Esp. jan./abr.). O gerenciamento de riscos em projetos gerenciados por abordagens ágeis: uma revisão sistemática da literatura

Tabela 2 - Principais características das abordagens tradicionais e ágeis

\begin{tabular}{|c|c|c|}
\hline Categorias & Abordagem Tradicionais & Abordagem Ágil \\
\hline Suposições fundamentais & $\begin{array}{l}\text { Sistemas totalmente especificados, } \\
\text { previsíveis e desenvolvido por meio } \\
\text { de um planejamento detalhado. }\end{array}$ & $\begin{array}{l}\text { Desenvolvido por times pequenos, } \\
\text { utiliza os princípios de desenho } \\
\text { contínuo da solução e baseado nos } \\
\text { feedbacks e mudanças. }\end{array}$ \\
\hline Estilo de gerenciamento & Comando e controle. & Liderança e colaboração. \\
\hline $\begin{array}{l}\text { Gerenciamento do } \\
\text { conhecimento }\end{array}$ & Explícito. & Tácito. \\
\hline Comunicação & Formal. & Informal. \\
\hline Modelo de desenvolvimento & Modelo de ciclo de vida (ex: cascata). & Modelo de entrega evolucionária. \\
\hline $\begin{array}{l}\text { Estrutura organizacional } \\
\text { ideal }\end{array}$ & $\begin{array}{l}\text { Mecanicista (burocrático e com alta } \\
\text { formalização). Direcionado para } \\
\text { grandes empresas. }\end{array}$ & $\begin{array}{l}\text { Orgânica (flexível, participativa e } \\
\text { colaborativa). Direcionada para } \\
\text { pequenas e médias empresas. }\end{array}$ \\
\hline Controle de qualidade & $\begin{array}{l}\text { Planejamento detalhado e controle } \\
\text { minucioso. }\end{array}$ & $\begin{array}{l}\text { Controle contínuo dos requerimentos, } \\
\text { desenho e solução. }\end{array}$ \\
\hline
\end{tabular}

Fonte: Adaptado de Dybå e Dingsøyr (2008).

Conforme sintetizado na Tabela 3, existem diversas metodologias com base no manifesto ágil, sendo que todas essas metodologias endereçam os desafios de um ambiente dinâmico e imprevisível, enfatizando as pessoas e sua criatividade sobre os processos. Vale destacar que, antes da publicação do Manifesto Ágil, já existiam algumas abordagens metodológicas, como a Extreme Programming (XP) e o Scrum.

Tabela 3 - Principais metodologias relacionadas com a abordagem ágil

\begin{tabular}{|c|c|c|}
\hline Metodologia & Características & Autores \\
\hline $\begin{array}{l}\text { Adaptive Software } \\
\text { Development } \\
\text { (ASD) }\end{array}$ & $\begin{array}{l}\text { Proposta por Highsmith (1997), essa abordagem foca na } \\
\text { colaboração e na auto-organização, sendo composta por três etapas } \\
\text { sobrepostas e não-lineares, a saber: especulação, colaboração e } \\
\text { aprendizado. Tem como característica iterações curtas e } \\
\text { incrementais, proximidade dos stakeholders, estrutura para evitar } \\
\text { o caos e desenvolvimento de aplicações em conjunto. }\end{array}$ & Jim Highsmith \\
\hline Crystal & $\begin{array}{l}\text { Composta por itens de diversos métodos, visto que seu criador } \\
\text { acredita na junção de diferentes características para viabilizar uma } \\
\text { metodologia ideal. Baseia-se em entregas incrementais e } \\
\text { cadenciadas dos produtos e na interação constante dos envolvidos. } \\
\text { Além disso, tem como princípio o conhecimento técnico mínimo } \\
\text { da equipe para suportar a demanda e assim garantir o sucesso do } \\
\text { projeto (Cockburn, 2004). }\end{array}$ & Alistar Cockburn \\
\hline Dynamic System & Objetiva entregas constantes por meio de um desenvolvimento & Grupo de \\
\hline Development & rápido, iterativo e incremental. Originalmente baseado no Rapid & especialistas \\
\hline Method (DSDM) & $\begin{array}{l}\text { Application Development (RAD), o DSDM foi concebido por um } \\
\text { grupo de especialistas e consultores do Reino Unido na década de } \\
\text { 1990. Consiste em três fases: pré-projeto, ciclo de vida e pós- } \\
\text { projeto (Stapleton \& Constable, 1997). }\end{array}$ & britânicos \\
\hline $\begin{array}{l}\text { Extreme } \\
\text { Programming (XP) }\end{array}$ & $\begin{array}{l}\text { O trabalho seminal sobre o tema foi escrito em 1999. Emprega uma } \\
\text { abordagem orientada a objetos como seu paradigma de } \\
\text { desenvolvimento preferido e envolve um conjunto de regras e } \\
\text { práticas constantes no contexto de quatro atividades metodológicas }\end{array}$ & Kent Beck \\
\hline
\end{tabular}


Fernandes, P. J. A., \& Rabechini, R. Jr. (2021, Ed. Esp. jan./abr.). O gerenciamento de riscos em projetos gerenciados por abordagens ágeis: uma revisão sistemática da literatura

\begin{tabular}{|c|c|c|}
\hline Metodologia & Características & Autores \\
\hline & $\begin{array}{l}\text { iterativas: Planejamento, Projeto, Codificação e Testes. O autor } \\
\text { define um conjunto de cinco valores que estabelecem as bases para } \\
\text { todo trabalho realizado como parte da XP: Comunicação, } \\
\text { Simplicidade, Feedback, Coragem e Respeito (Beck, 1999; } \\
\text { Pressman, 2011). }\end{array}$ & \\
\hline $\begin{array}{l}\text { Feature Driven } \\
\text { Development } \\
\text { (FDD) }\end{array}$ & $\begin{array}{l}\text { Influenciado pelo modelo de Peter Coad, o FDD é definido como } \\
\text { um processo iterativo e incremental de desenvolvimento de } \\
\text { software. Criado na década de } 1990 \text { por Jeff de Luca, essa } \\
\text { metodologia é composta por cinco processos: desenvolver um } \\
\text { modelo geral, construir uma lista de recursos, planejar por recursos, } \\
\text { desenhar por recurso e construir por recurso (Palmer \& Felsing, } \\
\text { 2002). }\end{array}$ & Jeff de Luca \\
\hline Lean Software & Criado pelo casal Mary e Tom Poppendieck em 2003, o LSD & Mary \\
\hline Development & baseia-se no sistema Lean originado na Toyota. Assim, objetiva a & Poppendieck e \\
\hline (LSD) & $\begin{array}{l}\text { eliminação dos desperdícios, melhoria da qualidade e otimização } \\
\text { de todo o processo (Poppendieck \& Poppendieck, 2003). }\end{array}$ & Tom Poppendieck \\
\hline Scrum & $\begin{array}{l}\text { Concebido inicialmente em } 1990 \text { por Jeff Sutherland, evoluiu } \\
\text { posteriormente com a contribuição de Ken Schwaber e Mike }\end{array}$ & $\begin{array}{l}\text { Jeff Sutherland, } \\
\text { Ken Schwaber }\end{array}$ \\
\hline & Beedle. Os princípios do Scrum são consistentes com o manifesto & e Mike Beedle \\
\hline & ágil e são usados para orientar as atividades de desenvolvimento & \\
\hline & dentro de um processo iterativo, onde cada ciclo do processo é & \\
\hline & $\begin{array}{l}\text { chamado de Sprint. Enfatiza o uso de um conjunto de padrões de } \\
\text { processos de software que provaram serem eficazes para projetos }\end{array}$ & \\
\hline & $\begin{array}{l}\text { com prazos de entrega apertados e requisitos críticos de negócio } \\
\text { (Pressman, 2011). }\end{array}$ & \\
\hline
\end{tabular}

Fonte: Elaborado pelos autores.

O método Scrum, desenvolvido principalmente por Ken Schwaber e Jeff Sutherland com base em suas experiências empíricas relacionadas a desenvolvimento de software, é uma das abordagens com filosofia ágil mais utilizadas pelas organizações. Além disso, estudos demonstram que o método Scrum vem ganhando popularidade no gerenciamento de projetos relacionados a outros contextos, além do desenvolvimento de software (Conforto et al., 2014; Moe et al., 2010).

\section{Procedimentos metodológicos}

Este artigo é uma análise exploratória, por meio de um estudo bibliométrico e uma revisão sistemática da literatura para delineamento dos temas: gerenciamento de riscos e abordagem ágil. Os artigos utilizados para este trabalho foram pesquisados em bases de dados relevantes para a área de Ciências Sociais Aplicadas, sendo utilizados termos em inglês, visto que as publicações de maior fator de impacto na área de Ciências Sociais Aplicadas estão neste idioma (Maccari \& Nishimura, 2014).

\subsection{Estudo bibliométrico}

A utilização da abordagem ágil para gerenciar projetos cresceu exponencialmente nos últimos anos, estimulando muito estudos sobre esse fenômeno, assim como o sucesso e as falhas dos projetos gerenciados por tal abordagem (Serrador \& Pinto, 2015). A avaliação de um fenômeno é importante para prover respostas aos problemas diários e 
Fernandes, P. J. A., \& Rabechini, R. Jr. (2021, Ed. Esp. jan./abr.). O gerenciamento de riscos em projetos gerenciados por abordagens ágeis: uma revisão sistemática da literatura

aumentar o conhecimento das organizações (Doh, 2015).

Zupic e Cater (2015) enfatizam a possibilidade de entender um determinado fenômeno e sua evolução, por meio de estudos acadêmicos já desenvolvidos sobre o tema em questão, possibilitando a identificação de sua estrutura intelectual e base científica.

Com isso, optou-se por iniciar este trabalho realizando um estudo bibliométrico, este que permite identificar os padrões, tendências e gerar visões sobre as informações e conhecimentos produzidos em uma determinada área, por meio das publicações, autores envolvidos e abordagens propostas, ilustrando assim seus agrupamentos e possíveis interações (Callon \& Courtial, 1995).

As principais ferramentas do estudo bibliométrico são as análises de citação e cocitação. A primeira considera os conteúdos produzidos e utilizados para referenciar outras pesquisas, sugerindo que artigos com um maior número de citação são mais influentes quando comparados ao demais com menos citações. Por outro lado, a cocitação ocorre quando artigos são citados em conjunto em outras pesquisas, possibilitando a avaliação do grau de ligação entre eles, revelando similaridade de conteúdo, embasamento em uma mesma teoria, padrões e associação entre autores (Ramos-Rodríguez \& Ruíz-Navarro, 2004).

A Figura 1, apresenta os passos executados neste estudo bibliométrico. Destacase que a pesquisa foi composta por uma análise de citação, com o objetivo de medir a frequência com que as referências são citadas, seguida de uma análise de cocitação, identificando pares de artigos citados simultaneamente. Para a elaboração da expressão de busca, foram consultados dois pesquisadores especialistas, sendo o primeiro em abordagem ágil e o segundo em gerenciamento de riscos.

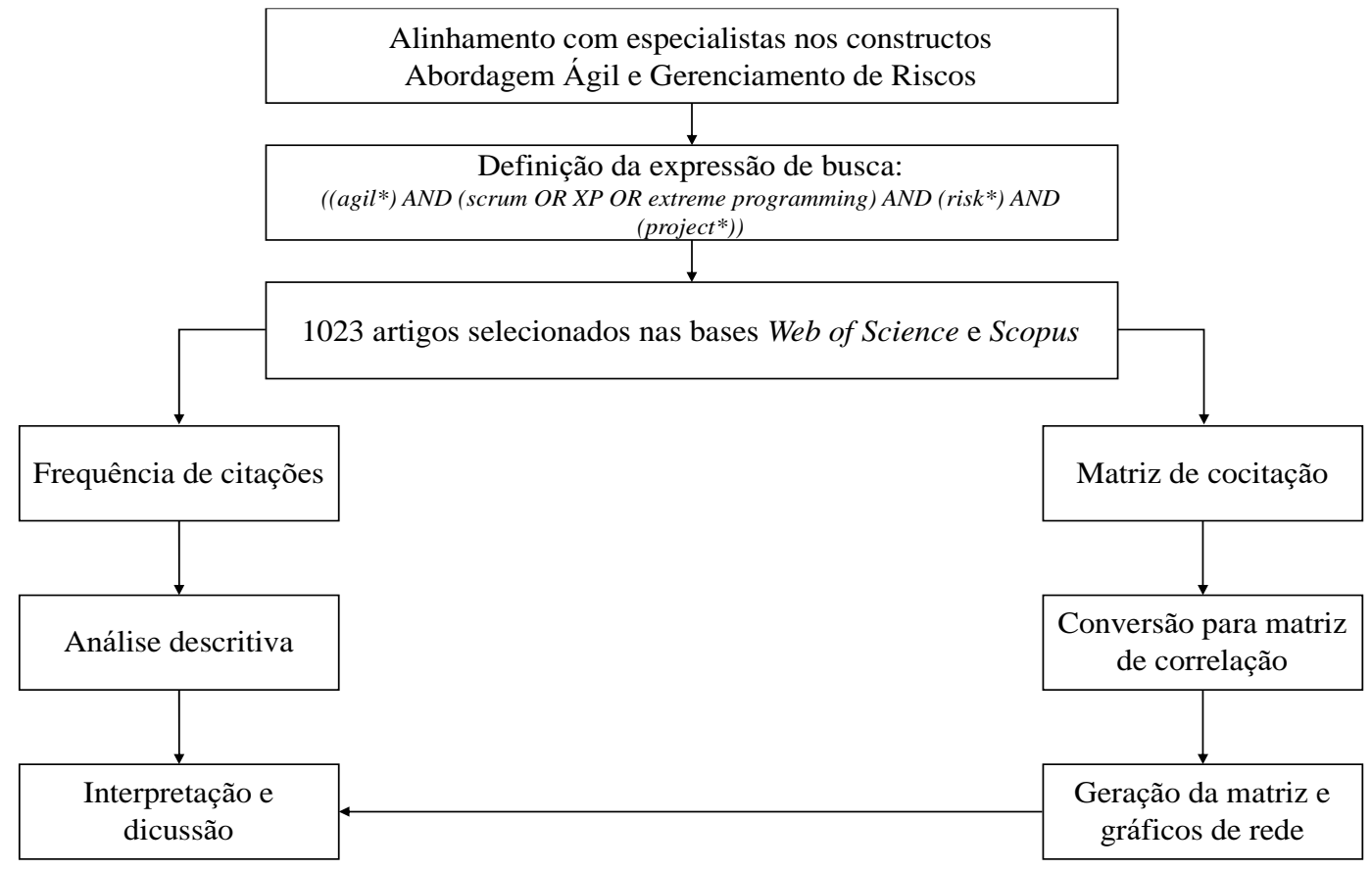

Figura 1 - Passos executados no estudo bibliométrico.

Fonte: Adaptado de Ramos-Rodríguez e Ruíz-Navarro (2004). 
Constatou-se que os termos 'agil*', 'risk' e 'project*' deveriam fazer parte da expressão de busca, mantendo-se o asterisco, visto que esse caractere permite a busca para qualquer derivação do radical. Com o intuito de não selecionar apenas estudos com foco no sucesso de projetos, entendeu-se que nenhuma expressão relacionada ao sucesso dos projetos deveria ser incluída na expressão de busca. Assim, a amostra contemplou todos os estudos que relacionavam gerenciamento de riscos e abordagens ágeis.

Por fim, um dos especialistas comentou que as pesquisas envolvendo abordagem ágil, muitas vezes são referenciadas pelos métodos pertencentes a esta abordagem. Assim, concluiu-se que os termos 'scrum', 'extreme programming' e ' $X P$ ' também deveriam ser incluídos na expressão, dado que representam os dois métodos da abordagem ágil mais utilizados, sendo ' $X P$ ' a abreviação de 'Extreme Programming' (Chow \& Cao, 2008).

Devido à relevância na área de Ciências Sociais Aplicadas, os dados para este estudo bibliométrico foram coletados das bases ISI Web of Science e Scopus. Para a realização das análises, necessitou-se de uma padronização das referências capturadas, visto que algumas delas apresentavam inconsistências relacionadas ao nome dos autores, volumes, número, páginas e nome dos periódicos.

\subsection{Revisão sistemática da literatura}

A segunda etapa deste trabalho foi a realização de uma revisão sistemática da literatura, com o objetivo de compilar as características dos modelos de gerenciamento de riscos em projetos gerenciados por abordagens ágeis. A condução de uma revisão sistemática de literatura se faz necessária, pois suas técnicas permitem mapear detalhadamente a literatura em uma determinada área de pesquisa, identificando os principais autores e a evolução do conhecimento sobre o tema ao longo do tempo (Petticrew \& Roberts, 2008).

Esta revisão sumariza a literatura relacionada a gerenciamento de riscos, focando na identificação da forma de aplicação deste tema em projetos gerenciados por abordagens ágeis, validando o sucesso e mapeando os principais riscos envolvidos nesses projetos. A revisão sistemática da literatura é relevante para a área acadêmica, visto que desenvolve a base de conhecimento e auxiliar na resposta das questões de pesquisa (Tranfield et al., 2003). Assim, foram definidos 10 passos, conforme detalhamento abaixo:

(1) Definição da questão de pesquisa e expressão de busca, acrescentando a variável sucesso na expressão definida anteriormente para o estudo bibliométrico:

( agil* OR scrum OR xp OR "extreme programming") AND ( risk*) AND (project*) AND (success*));

(2) Composição do comitê utilizado para validar a expressão de busca, composto por dois pesquisadores, sendo um deles especialista em gerenciamento de 
riscos e outro especialista em abordagens ágeis;

(3) Definição do protocolo de pesquisa, considerando as ferramentas usadas para acessar as informações nas bases de dados (Web of Science e Scopus); itens considerados na busca (título, palavras-chave e resumo); tipo de documento (artigos); e relação do artigo com os temas (gerenciamento de projetos, gerenciamento de riscos, sucesso de projetos e abordagens ágeis);

(4) Considerando as palavras mencionadas no passo 1, a pesquisa retornou 332 publicações;

(5) O filtro aplicado no tipo de documento (artigos) resultou em 84 estudos;

(6) As análises dos resumos e da relação do estudo com os temas (gerenciamento de projetos, gerenciamento de riscos, sucesso de projetos e abordagens ágeis), resultou em um montante de 16 artigos;

(7) Extração dos dados para a análise qualitativa de conteúdo;

(8) Os casos selecionados foram complementados com a literatura existente, embasando as análises e decisões;

(9) Avaliação das limitações, vieses, riscos e categorias;

(10) Emissão do relatório final.

Destaca-se que os passos mencionados foram baseados nas sugestões de Petticrew e
Roberts (2008), seguindo todo o rigor metodológico sugerido por esses autores.

\section{Resultados}

Conforme descrito na Tabela 4, considerando os resultados obtidos na pesquisa bibliométrica, verificou-se que $55 \%$ das publicações foram realizadas em conferências ou congressos, demonstrando a existência de poucos estudos publicados em periódicos acadêmicos envolvendo os constructos ‘Abordagem Ágil' e 'Gerenciamento de Riscos'.

De acordo com González-Albo e Bordons (2011), os estudos publicados em conferências ou congressos, denominados em inglês como proceedings papers, diferenciamse dos estudos publicados em periódicos, conhecidos no mundo acadêmico como 'artigos'. Entretanto, os autores enfatizam a complementariedade desses dois tipos de estudos.

Os proceedings papers são estudos em sua fase inicial, possibilitando que seus pesquisadores, por meio das conferências e congressos, capturem possíveis críticas e futuramente evoluam seus estudos. Por outro lado, os artigos publicados em periódicos, geralmente são mais completos e maduros (Montesi \& Owen, 2008). Os autores complementam que, geralmente, os proceeding papers são mais sucintos, ou seja, contém somente os itens mais relevantes do estudo, enquanto os artigos tendem a ser mais detalhados, permitindo sua replicação e 
Fernandes, P. J. A., \& Rabechini, R. Jr. (2021, Ed. Esp. jan./abr.). O gerenciamento de riscos em projetos gerenciados por abordagens ágeis: uma revisão sistemática da literatura

completo entendimento. Por fim, concluem que

posteriormente evoluídos e publicados em $33 \%$ dos proceedings papers são periódicos.

Tabela 4 - Distribuição das publicações

\begin{tabular}{lcc}
\hline Tipo de Publicação & Quantidade de publicações & $\%$ da amostra \\
\hline Conferências e Congressos & 563 & $55 \%$ \\
Revistas não acadêmicas & 302 & $30 \%$ \\
Periódicos acadêmicos & 158 & $15 \%$ \\
\hline
\end{tabular}

Fonte: Elaborado pelos autores.

Considerando os estudos publicados em periódicos, dado que esses são mais completos e maduros, nota-se que da amostra de periódicos, $50 \%$ possuem classificação A1, considerando a avaliação do 'QUALIS', modelo criado pela Coordenação de Aperfeiçoamento de Pessoal de Nível Superior (CAPES), ou seja, são considerados periódicos com alto fator de impacto, mostrando a relevância dos temas para o mundo acadêmico.

No Brasil, os periódicos científicos são também classificados pela avaliação QUALIS, essa que considera principalmente o Fator de Impacto ISI (J) e o Índice H SCImago (H) como balizadores para a definição da avaliação. Os periódicos podem ser classificados em sete estratos: A1, A2, B1, B2, B3, B4, B5 e C, sendo o estrato A1 o mais relevante e o estrato $\mathrm{C}$ a classificação com menor impacto (Lorenzini Erdmann et al., 2009). Destaca-se que, em 2019, a CAPES definiu uma nova proposta para o sistema de avaliação, alterando alguns conceitos de avaliação e a estratificação dos periódicos (A1, A2, A3, A4, B1, B2, B3 e B4). Entretanto, essas alterações ainda não foram completamente implementadas até a conclusão desta pesquisa (Costa et al., 2020). Por este motivo, as informações aqui apresentadas foram baseadas nas diretrizes vigentes, citadas anteriormente. A Figura 2 mostra a evolução das pesquisas selecionadas por ano de publicação, considerando todos os estudos da amostra selecionada. Destaca-se o acréscimo de publicações após a divulgação do manifesto ágil em 2001, disseminando a abordagem ágil tanto no setor acadêmico quanto no setor profissional.

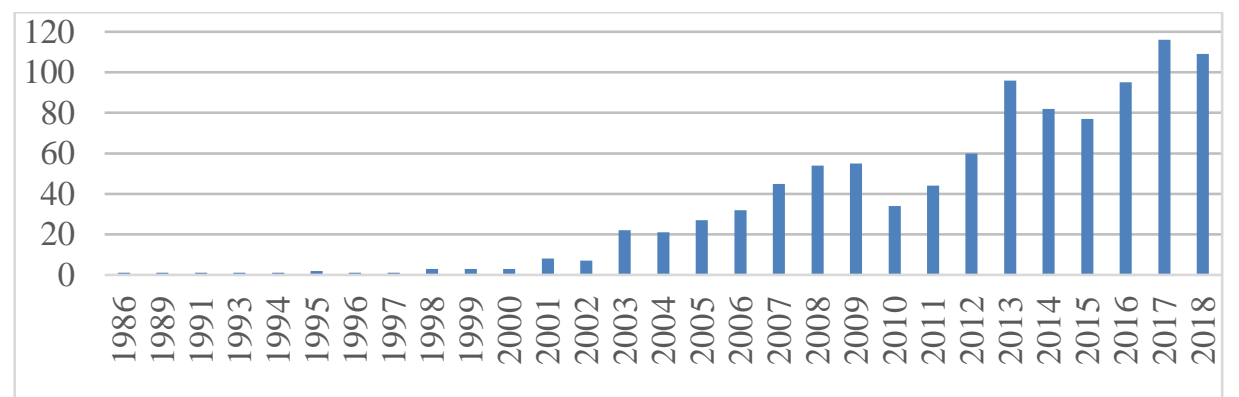


Fernandes, P. J. A., \& Rabechini, R. Jr. (2021, Ed. Esp. jan./abr.). O gerenciamento de riscos em projetos gerenciados por abordagens ágeis: uma revisão sistemática da literatura

Figura 2 - Evolução das publicações selecionadas para este estudo.

Fonte: Elaborado pelos autores.

Considerando todas as 1023 pesquisas capturadas nas bases de dados, realizou-se a análise de citação, por meio do software Bibexcel. Assim, foi possível identificar as pesquisas mais representativas, observando a frequência de citações com que outros trabalhos as referenciaram. A Tabela 5 apresenta as referências citadas trinta vezes ou mais, caracterizando-se as referências mais relevantes sobre os temas pesquisados.

Nota-se que algumas pesquisas citaram versões diferentes de uma mesma referência, como por exemplo as citações de Beck (1999, 2000), demonstrando a relevância de cada uma das versões para a composição dos resultados.

Tabela 5 - Frequência de citação dos artigos usados nas referências

\begin{tabular}{|c|c|c|c|c|}
\hline Referência & $\begin{array}{c}\text { Tipo de } \\
\text { publicação }\end{array}$ & Proposta & $\begin{array}{l}\text { Quantidade } \\
\text { de citações }\end{array}$ & $\begin{array}{c}\% \text { da } \\
\text { amostra }\end{array}$ \\
\hline (Beck, 2001) & Website & Conceitos & 70 & $0,3 \%$ \\
\hline (Beck, 2000) & Livro & Conceitos & 62 & $0,2 \%$ \\
\hline (Beck, 1999) & Livro & Conceitos & 58 & $0,2 \%$ \\
\hline (Schwaber et al., 2004) & Livro & Conceitos & 58 & $0,2 \%$ \\
\hline (Abrahamsson, 2002) & Livro & Resultados & 45 & $0,2 \%$ \\
\hline (Dybå \& Dingsøyr, 2008) & Artigo & Resultados & 43 & $0,2 \%$ \\
\hline (Schwaber et al., 2002) & Livro & Conceitos & 43 & $0,2 \%$ \\
\hline (Boehm, 1991) & Artigo & Modelo & 41 & $0,2 \%$ \\
\hline (Cockburn \& Highsmith, 2001) & Artigo & Resultados & 40 & $0,2 \%$ \\
\hline (PMI, 2013) & Livro & Conceitos & 40 & $0,2 \%$ \\
\hline (Boehm, 2002) & Artigo & Conceitos & 37 & $0,1 \%$ \\
\hline (Boehm et al., 2003) & Livro & Conceitos & 37 & $0,1 \%$ \\
\hline (Beck, 2004) & Livro & Conceitos & 36 & $0,1 \%$ \\
\hline (Boehm \& Turner, 2003) & Artigo & Modelo & 34 & $0,1 \%$ \\
\hline (Boehm et al., 2004) & Livro & Conceitos & 33 & $0,1 \%$ \\
\hline (Cockburn, 2002) & Livro & Conceitos & 33 & $0,1 \%$ \\
\hline (Highsmith, 2002) & Livro & Conceitos & 33 & $0,1 \%$ \\
\hline (Chow \& Cao, 2008) & Artigo & Resultados & 32 & $0,1 \%$ \\
\hline (Larman \& Basili, 2003) & Artigo & Conceitos & 32 & $0,1 \%$ \\
\hline (Poppendieck \& Poppendieck, 2003) & Livro & Conceitos & 32 & $0,1 \%$ \\
\hline (Nerur et al., 2005) & Artigo & Resultados & 30 & $0,1 \%$ \\
\hline
\end{tabular}


Fernandes, P. J. A., \& Rabechini, R. Jr. (2021, Ed. Esp. jan./abr.). O gerenciamento de riscos em projetos gerenciados por abordagens ágeis: uma revisão sistemática da literatura

\begin{tabular}{lcccc}
\hline Referência & $\begin{array}{c}\text { Tipo de } \\
\text { publicação }\end{array}$ & Proposta & $\begin{array}{c}\text { Quantidade } \\
\text { de citações }\end{array}$ & $\begin{array}{c}\% \text { da } \\
\text { amostra }\end{array}$ \\
\hline Totais & & 869 & $3,4 \%$ \\
\hline
\end{tabular}

Fonte: Elaborado pelos autores.

O resultado da análise de citação dos artigos mais citados revela que 57\% das publicações são livros sobre o tema em questão, frente a $38 \%$ de publicações em periódicos acadêmicos, destacando a importância de uma linguagem mais próxima à realidade das organizações. Além disso, aponta que $67 \%$ das publicações abordam os conceitos da abordagem ágil, atestando que este tema é um fenômeno recente, sendo necessário contextualizar antes de realizar estudos empíricos. Verificou-se ainda que apenas duas das publicações abordam claramente o os riscos envolvidos na abordagem ágil ou os processos relacionados ao gerenciamento de riscos (Boehm, 1991; Boehm \& Turner, 2003), demonstrando a necessidade de estudos que avaliem 'Abordagem Ágil' e 'Gerenciamento de Riscos' simultaneamente. Corroborando com essa assertiva, Carvalho e Rabechini Jr. (2014), por meio de uma revisão da literatura, identificaram as principais publicações relacionadas a gerenciamento de riscos, contendo apenas a pesquisa de Boehm (1991) nessa lista, ou seja, entende-se que as publicações relacionadas à abordagem ágil pouco citam as principais referência sobre gestão de riscos. Para a análise de cocitação, conforme Tabela 6, gerou-se uma matriz com auxílio do software Bibexcel, apontando as publicações com algum grau de ligação entre elas, visto que foram citadas simultaneamente por outros estudos. As publicações com maior grau de ligação foram representadas por numerais em negrito, sendo ainda enfatizadas com preenchimento cinza, as publicações com os graus mais altos.

Tabela 6 - Matriz de cocitação 
Fernandes, P. J. A., \& Rabechini, R. Jr. (2021, Ed. Esp. jan./abr.). O gerenciamento de riscos em projetos gerenciados por abordagens ágeis: uma revisão sistemática da literatura

\begin{tabular}{|l|c|cccccccccccccccccccc|c|}
\hline \multicolumn{1}{l|}{} & & $\mathrm{A}$ & $\mathrm{B}$ & $\mathrm{C}$ & $\mathrm{D}$ & $\mathrm{E}$ & $\mathrm{F}$ & $\mathrm{G}$ & $\mathrm{H}$ & $\mathrm{I}$ & $\mathrm{J}$ & $\mathrm{K}$ & $\mathrm{L}$ & $\mathrm{M}$ & $\mathrm{N}$ & $\mathrm{O}$ & $\mathrm{P}$ & $\mathrm{Q}$ & $\mathrm{R}$ & $\mathrm{S}$ & $\mathrm{T}$ & $\mathrm{T}$ Total \\
\hline (Boehm, 2002) & $\mathrm{G}$ & 1 & 1 & 2 & $\mathbf{3}$ & 2 & 0 & & 2 & 0 & 1 & 0 & $\mathbf{5}$ & 1 & $\mathbf{4}$ & $\mathbf{3}$ & 1 & 1 & 0 & 1 & 0 & 28 \\
(Boehm et al., 2003) & $\mathrm{H}$ & 0 & $\mathbf{3}$ & 1 & 1 & 0 & 0 & 2 & & 0 & 0 & 0 & 0 & $\mathbf{3}$ & 2 & 0 & 0 & 1 & 0 & 1 & 0 & 14 \\
(Boehm et al., 2004) & $\mathrm{I}$ & 1 & 0 & 2 & $\mathbf{3}$ & 0 & 0 & 0 & 0 & & $\mathbf{3}$ & $\mathbf{5}$ & $\mathbf{5}$ & 1 & 2 & 0 & 1 & 2 & 0 & 0 & 0 & 25 \\
(Chow \& Cao, 2008) & $\mathrm{J}$ & 0 & 1 & $\mathbf{3}$ & 2 & 1 & 0 & 1 & 0 & $\mathbf{3}$ & & $\mathbf{3}$ & 1 & $\mathbf{5}$ & 2 & $\mathbf{3}$ & 0 & 1 & 1 & $\mathbf{3}$ & 0 & 30 \\
(Cockburn \& Highsmith, & $\mathrm{K}$ & 2 & 0 & 2 & $\mathbf{3}$ & 2 & 1 & 0 & 0 & $\mathbf{5}$ & $\mathbf{3}$ & & 1 & 1 & 2 & 0 & 1 & 2 & 0 & 0 & 0 & 25 \\
2001) & $\mathrm{L}$ & 1 & 0 & 1 & 1 & 1 & 1 & $\mathbf{5}$ & 0 & $\mathbf{5}$ & 1 & 1 & & 0 & $\mathbf{4}$ & 2 & 1 & $\mathbf{4}$ & 0 & 0 & 0 & 28 \\
(Cockburn, 2002) & $\mathrm{M}$ & 1 & 2 & $\mathbf{5}$ & 1 & 1 & 1 & 1 & $\mathbf{3}$ & 1 & $\mathbf{5}$ & 1 & 0 & & 2 & $\mathbf{3}$ & 0 & 2 & 0 & $\mathbf{5}$ & 0 & 34 \\
(Dybå \& Dingsøyr, 2008) & $\mathrm{N}$ & 1 & 1 & 0 & $\mathbf{4}$ & 2 & $\mathbf{4}$ & $\mathbf{4}$ & 2 & 2 & 2 & 2 & $\mathbf{4}$ & 2 & & 1 & 1 & $\mathbf{3}$ & 0 & $\mathbf{3}$ & 0 & 38 \\
(Highsmith, 2002) & $\mathrm{O}$ & 0 & 1 & 2 & 1 & 0 & 2 & $\mathbf{3}$ & 0 & 0 & $\mathbf{3}$ & 0 & 2 & $\mathbf{3}$ & 1 & & 1 & 1 & 0 & 1 & 0 & 21 \\
(Larman \& Basili, 2003) & $\mathrm{P}$ & 0 & 0 & 1 & 0 & 1 & 0 & 1 & 0 & 1 & 0 & 1 & 1 & 0 & 1 & 1 & & 0 & 0 & 1 & 0 & 9 \\
(Nerur et al., 2005) & $\mathrm{Q}$ & 0 & 0 & 1 & 1 & 1 & 2 & 1 & 1 & 2 & 1 & 2 & $\mathbf{4}$ & 2 & $\mathbf{3}$ & 1 & 0 & 0 & 1 & 0 & 23 \\
(PMI, 2013) & $\mathrm{R}$ & 0 & 0 & 0 & 0 & 0 & 0 & 0 & 0 & 0 & 1 & 0 & 0 & 0 & 0 & 0 & 0 & 0 & 0 & 0 & 1 \\
(Schwaber et al., 2002) & $\mathrm{S}$ & 0 & 2 & $\mathbf{5}$ & 2 & 0 & 1 & 1 & 1 & 0 & $\mathbf{3}$ & 0 & 0 & $\mathbf{5}$ & $\mathbf{3}$ & 1 & 1 & 1 & 0 & 0 & 26 \\
(Schwaber et al., 2004) & $\mathrm{T}$ & 0 & 0 & 1 & 0 & 0 & 0 & 0 & 0 & 0 & 0 & 0 & 0 & 0 & 0 & 0 & 0 & 0 & 0 & 0 & 1 \\
\hline
\end{tabular}

Fonte: Elaborado pelos autores.

Gerada por meio do software VOSviewer e baseada na coluna 'Total' da matriz citada previamente, a Figura 3 apresenta graficamente a rede de cocitação com grau de relação superior a dez e segregado em grupos, representados pelas cores dos nós, a saber: (1) vermelho: artigos com objetivo de contextualizar as abordagens ágeis; (2) verde: artigos com foco nas técnicas das abordagens ágeis e; (3) azul: artigos relacionados aos riscos das técnicas das abordagens ágeis. 
Aprovado em: 21 jan. 2021

Edição Especial: Do manifesto ágil à agilidade organizacional

Editora Chefe: Rosária de Fátima Segger Macri Russo

Editor Científico: Luciano Ferreira da Silva

Editor Convidado: Claudio Luis Carvalho Larieira

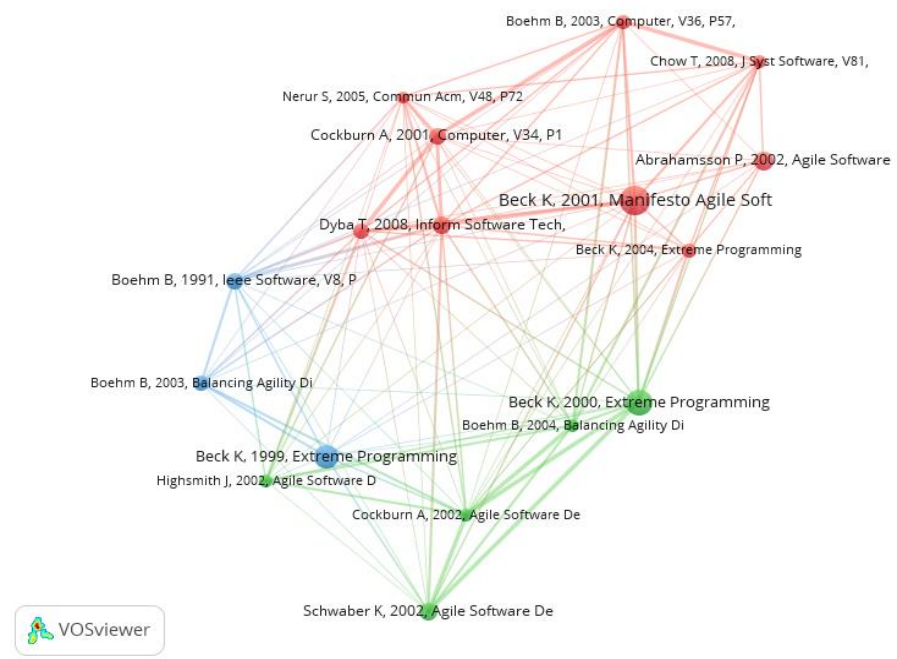

Figura 3 - Rede de cocitação.

Fonte: Elaborado pelos autores.

Considerando o tamanho dos nós da Figura 3, destaca-se que a referência com maior número de citações está relacionada ao manifesto ágil, este que foi responsável pela popularização das abordagens ágeis.

Identificou-se que a revisão sistemática da literatura sobre os estudos empíricos relacionados à abordagem ágil, realizada por Dybå e Dingsøyr (2008), foi a pesquisa com maior quantidade de relações, mostrando a necessidade de informações empíricas para embasar os estudos relacionados à abordagem ágil. Com relação às publicações focadas em explorar os conceitos da abordagem ágil, verificou-se que as obras de Beck (2000), Boehm et al. (2004), Cockburn (2002) e Schwaber et al (2002) são complementares, haja visto que foram referenciadas diversas vezes em conjunto.Considerando a soma das relações de cocitação contidas na coluna 'Total' da Tabela 6, constatou-se que a publicação de Boehm (1991), esta que aborda os princípios do gerenciamento de riscos, possui baixa relação com os demais estudos, ratificando a necessidade de pesquisas envolvendo ambos os constructos, 'Abordagem Ágil' e 'Gerenciamento de Riscos'. Por fim, detectouse forte relação entre o estudo de Chow e Cao (2008), cujo objetivo é mapear os fatores críticos de sucesso em projetos gerenciados por abordagem ágil, e o estudo de Boehm e Turner (2003), este que explora os riscos envolvidos em projetos gerenciados pela abordagem ágil, demonstrando relação entre os constructos e, justificando estudos envolvendo esses dois temas.

Complementando o estudo bibliométrico, realizou-se a revisão sistemática da literatura, selecionando 16 artigos para análise de conteúdo, conforme Tabela 7 . Ressalta-se que os artigos foram selecionados com base no título e leitura prévia do resumo.

Os artigos foram divulgados no período de 2010 a 2018, sendo a maioria deles publicada 
Fernandes, P. J. A., \& Rabechini, R. Jr. (2021, Ed. Esp. jan./abr.). O gerenciamento de riscos em projetos gerenciados por abordagens ágeis: uma revisão sistemática da literatura

em periódicos com alto fator de impacto, considerando o fator Qualis. Além disso, predominam os artigos com abordagens qualitativa e ensaio teórico, atestando que o gerenciamento de riscos em projetos gerenciados por abordagens ágeis é um fenômeno recente, sem muitas pesquisas empíricas sobre este tema (Creswell, 2010).

Enfatiza-se que todos os artigos avaliados consideram projetos de tecnologia como unidade de análise, sendo projetos para desenvolvimento de software ou outra finalidade relacionada à tecnologia, demonstrando que apesar da expansão citada por alguns autores, tais como Serrador e Pinto (2015), as abordagens ágeis estão predominantemente presentes em projetos de tecnologia.

Tabela 7 - Artigos selecionados para a revisão sistemática da literatura

\begin{tabular}{lcll}
\hline Referência & Qualis & Abordagem & Unidade de Análise \\
\hline (Chen et al., 2016) & A1 & Simulação & Projeto de Software \\
(da Silva \& Neto, 2015) & B1 & Quantitativo & Projeto de Software \\
(Donmez \& Grote, 2018) & A1 & Qualitativo & Projeto de Software \\
(Ghobadi \& Mathiassen, 2017) & A1 & Qualitativo e Simulação & Projeto de Software \\
(Howell et al., 2010) & A1 & Ensaio Teórico & Projeto de Software \\
(Lee \& Baby, 2013) & B1 & Qualitativo & Projeto de Tecnologia \\
(Mishra et al., 2017) & A2 & Métodos Mistos & Projeto de Tecnologia \\
(Mousaei \& Javdani, 2018) & B2 & Ensaio Teórico e Quantitativo & Projeto de Software \\
(Odzaly et al., 2018) & B1 & Ensaio Teórico & Projeto de Software \\
(Perkusich et al., 2017) & B2 & Simulação & Projeto de Software \\
(Shmueli \& Ronen, 2017) & A1 & Ensaio Teórico & Projeto de Software \\
(Shrivastava \& Rathod, 2014) & A2 & Revisão Sistemática da Literatura & Projeto de Software \\
(Shrivastava \& Rathod, 2015) & A1 & Qualitativo & Projeto de Software \\
(Shrivastava \& Rathod, 2017) & A1 & Quantitativo e Ensaio Teórico & Projeto de Software \\
(Sundararajan et al., 2014) & B1 & Qualitativo & Projeto de Software \\
(Wanderley et al., 2015) & B1 & Ensaio Teórico & Projeto de Software \\
\hline
\end{tabular}

Fonte: Elaborado pelos autores.

Constatou-se ainda a inexistência de um modelo padrão para o gerenciamento de riscos em projetos gerenciados por abordagens ágeis, visto que o principal objetivo de $63 \%$ dos artigos avaliados é justamente a proposição de um novo modelo para gerenciamento dos riscos em projetos com as características citadas, conforme apresentado resumidamente na Tabela 8. Os artigos não apresentados nesta tabela não tinham como objetivo propor um modelo de gerenciamento de riscos ou baseavam seus estudos em modelos já consolidados na literatura, ou seja, modelos que 
Fernandes, P. J. A., \& Rabechini, R. Jr. (2021, Ed. Esp. jan./abr.). O gerenciamento de riscos em projetos gerenciados por abordagens ágeis: uma revisão sistemática da literatura

foram criados inicialmente para projetos

gerenciados por abordagens tradicionais.

Tabela 8 - Modelos propostos nos artigos avaliados

\begin{tabular}{|c|c|c|}
\hline Referência & Base do Modelo & Objetivo do Modelo \\
\hline (Chen et al., 2016) & RAD (Rapid Application Development). & $\begin{array}{l}\text { Modelo de eficiência de custos e } \\
\text { gerenciamento de riscos no } \\
\text { desenvolvimento ágil de sistemas de } \\
\text { big data. }\end{array}$ \\
\hline $\begin{array}{l}\text { (Ghobadi \& Mathiassen, } \\
\text { 2017) }\end{array}$ & $\begin{array}{l}\text { Estratégia de risco e questões de } \\
\text { compartilhamento de conhecimento } \\
\text { identificadas na literatura. }\end{array}$ & $\begin{array}{l}\text { Modelo para gerenciar riscos de } \\
\text { compartilhamento de conhecimento } \\
\text { em projetos ágeis de software. }\end{array}$ \\
\hline (Howell et al., 2010) & Teoria de Contingência de Projetos. & $\begin{array}{l}\text { Modelo para gerenciar riscos em } \\
\text { projetos ágeis. }\end{array}$ \\
\hline (Lee \& Baby, 2013) & Princípios de SOA. & $\begin{array}{l}\text { Modelo para identificar e gerenciar } \\
\text { riscos em projetos globais de TI. }\end{array}$ \\
\hline (Mousaei \& Javdani, 2018) & Metodologias Scrum e Prince2. & $\begin{array}{l}\text { Modelo para gerenciar riscos em } \\
\text { projetos ágeis. }\end{array}$ \\
\hline (Odzaly et al., 2018) & $\begin{array}{l}\text { Goal-Driven Software Development Risk } \\
\text { Management Model (GSRM). }\end{array}$ & $\begin{array}{l}\text { Modelo para gerenciar riscos em } \\
\text { projetos ágeis. }\end{array}$ \\
\hline (Perkusich et al., 2017) & Redes bayesianas. & $\begin{array}{l}\text { Modelo para melhoria contínua de } \\
\text { projetos Scrum, incluindo o processo } \\
\text { de gerenciamento de riscos. }\end{array}$ \\
\hline (Shmueli \& Ronen, 2017) & $\begin{array}{l}\text { Problemas de desenvolvimento de software } \\
\text { identificados na literatura. }\end{array}$ & $\begin{array}{l}\text { Modelo para consolidar os problemas } \\
\text { do desenvolvimento de software. }\end{array}$ \\
\hline $\begin{array}{l}\text { (Shrivastava \& Rathod, } \\
\text { 2017) }\end{array}$ & $\begin{array}{l}\text { Estrutura para implementação da } \\
\text { metodologia Scrum no desenvolvimento } \\
\text { distribuído. }\end{array}$ & $\begin{array}{l}\text { Modelo para gerenciar riscos em } \\
\text { projetos ágeis distribuídos. }\end{array}$ \\
\hline (Wanderley et al., 2015) & $\begin{array}{l}\text { Ponto de risco, cujo objetivo é medir os } \\
\text { riscos no contexto do gerenciamento de } \\
\text { vários projetos de software como ferramenta } \\
\text { de suporte para os gerentes de projeto. }\end{array}$ & $\begin{array}{l}\text { Proposta de métrica de } \\
\text { gerenciamento de riscos para vários } \\
\text { projetos. }\end{array}$ \\
\hline
\end{tabular}

Fonte: Elaborado pelos autores.

Considerando os artigos avaliados e com o intuito de mapear os riscos relacionados a projetos gerenciados por abordagens ágeis, identificou-se os riscos reportados em cada artigo, sendo esses, posteriormente, classificados nas seguintes categorias: organizacional, pessoas, processos, técnico e projeto. Assim, verificou-se que a categoria 'pessoas' contempla a maior parte dos riscos reportados nos artigos avaliados, sendo 'comportamento dos stakeholders' considerado o principal risco, seguido de 'comunicação' e 'colaboração da equipe', conforme Tabela 9. Destaca-se que esta tabela possui apenas os riscos recorrentes, ou seja, mencionados em dois ou mais artigos, aumentando a assertividade na seleção dos riscos mais relevantes para projetos gerenciados por abordagens ágeis.

Tabela 9 - Riscos relacionados aos projetos gerenciados por abordagens ágeis

\begin{tabular}{llc}
\hline Risco & Categoria do Risco & Quantidade* \\
\hline Comportamento das partes interessadas & Pessoas & 9
\end{tabular}


Fernandes, P. J. A., \& Rabechini, R. Jr. (2021, Ed. Esp. jan./abr.). O gerenciamento de riscos em projetos gerenciados por abordagens ágeis: uma revisão sistemática da literatura

\begin{tabular}{|c|c|c|}
\hline Risco & Categoria do Risco & Quantidade* \\
\hline Comunicação & Pessoas & 8 \\
\hline Colaboração da equipe & Pessoas & 8 \\
\hline Requisitos pouco claros & Processos & 8 \\
\hline Gestão de mudanças & Processos & 7 \\
\hline Eficiência & Pessoas & 7 \\
\hline Localização & Projeto & 7 \\
\hline Habilidades técnicas & Técnico & 7 \\
\hline Cultura & Pessoas & 6 \\
\hline Infraestrutura e recursos & Projeto & 6 \\
\hline Priorização & Processos & 6 \\
\hline Gerenciamento da equipe & Processos & 6 \\
\hline Documentação & Técnico & 5 \\
\hline Compartilhamento de informações & Pessoas & 5 \\
\hline Idioma & Técnico & 5 \\
\hline Motivação & Pessoas & 5 \\
\hline Fuso horário & Projeto & 5 \\
\hline Confiança & Pessoas & 5 \\
\hline Empoderamento & Processos & 4 \\
\hline Conhecimento & Técnico & 4 \\
\hline Qualidade & Processos & 4 \\
\hline Práticas ágeis incorretas & Organizacional & 4 \\
\hline Gestão de contratos & Processos & 3 \\
\hline Estimativa de esforço & Projeto & 3 \\
\hline Escopo do projeto extenso & Projeto & 3 \\
\hline Resistência às práticas ágeis & Organizacional & 2 \\
\hline Rotatividade das pessoas & Organizacional & 2 \\
\hline Estrutura do processo & Processos & 2 \\
\hline Criação do termo de abertura & Projeto & 2 \\
\hline Capacidade da equipe & Pessoas & 2 \\
\hline Gestão de terceiros & Processos & 2 \\
\hline Custo com viagens & Organizacional & 2 \\
\hline
\end{tabular}

Conforme mencionado e apresentado na Tabela 9, verificou-se que a categoria 'Pessoas' contempla a maior quantidade dos riscos reportados nos artigos avaliados, destacando que se os riscos desta categoria não forem corretamente gerenciados podem impactar o sucesso dos projetos gerenciados por abordagens ágeis. Além disso, enfatiza-se que a categoria supracitada está diretamente relacionada a dois dos valores descritos para a abordagem ágil 'Indivíduos e interações mais que processos e ferramentas' e 'Colaboração com o cliente mais que negociação de contratos'. Em outras palavras, entendeu-se que apesar de ser um dos principais benefícios da abordagem ágil, as práticas relacionadas aos indivíduos e suas interações, se não gerenciadas e realizadas adequadamente, podem contribuir diretamente para o fracasso do projeto. Seguindo o mesmo raciocínio, a segunda categoria com mais riscos também está diretamente relacionada a dois dos valores da abordagem ágil 'Software em funcionamento mais que documentação abrangente' e 'Responder a mudanças mais que seguir um plano.

Por fim, destaca-se que as características dos projetos gerenciados por 
Fernandes, P. J. A., \& Rabechini, R. Jr. (2021, Ed. Esp. jan./abr.). O gerenciamento de riscos em projetos gerenciados por abordagens ágeis: uma revisão sistemática da literatura

abordagem ágil influenciam consideravelmente o sucesso destes, tais como as características dos projetos globais ou distribuídos, devido à localização distribuída de seus envolvidos, fuso-horário, cultura e linguagem.

\section{Conclusão}

Com base nos resultados do estudo bibliométrico, identificou-se que a relação entre o gerenciamento de riscos e a abordagem ágil é um fenômeno recente, considerando a concentração de pesquisas publicadas nos últimos cinco anos, assim como a baixa quantidade de estudos empíricos relacionando esses constructos e a discreta relação obtida na análise de cocitação. Além disso, a análise de citação revelou que muitos dos principais estudos foram apenas publicados em conferências e congressos, caracterizando pesquisas em sua fase inicial.

$$
\text { Complementando os itens }
$$

mencionados, a revisão sistemática da literatura demonstrou que o uso da abordagem ágil, de fato, identificou e eliminou diversos problemas oriundos da abordagem tradicional, tais como rigidez e linearidade dos projetos. Entretanto, originou outros problemas, visto que muitos dos riscos identificados nos projetos gerenciados pela referida abordagem, estão diretamente relacionados com os valores centrais do manifesto ágil. Em outras palavras, se os riscos oriundos dos principais benefícios da abordagem ágil, tais como interações, comunicação, colaboração e flexibilidade, não forem corretamente gerenciados podem contribuir diretamente para o fracasso dos projetos gerenciados por tal abordagem.
Destaca-se ainda que, além de não abordar projetos complexos, as pesquisas analisadas consideraram somente projetos de tecnologia como unidade de análise, corroborando que, possivelmente, a abordagem ágil seja indicada apenas para projetos com determinadas características. Dentre outros fatores, a quantidade de envolvidos contribui para a classificação de um projeto como complexo, devido aos desafios existentes no gerenciamento das comunicações deste projeto. Por sua vez, os projetos de tecnologia possuem uma certa padronização nos processos, possibilitando um maior controle de cada etapa do projeto.

Por fim, constatou-se que, baseando-se principalmente em modelos de gerenciamento de riscos originalmente criados para projetos gerenciados por abordagem tradicional, grande parte dos artigos avaliados tinham como objetivo propor um modelo para o gerenciamento de riscos em projetos gerenciados por abordagens ágeis, demonstrando a inexistência de um modelo já consolidado na literatura para essa finalidade.

As contribuições práticas deste estudo estão em demonstrar a importância do gerenciamento de riscos para o sucesso dos projetos gerenciados por abordagens ágeis, assim como identificar os principais riscos e modelos de gerenciamento de riscos utilizados em projetos com as características citadas. Sugere-se para pesquisas futuras, avaliações em outras bases de dados e, possivelmente, incluindo novos termos na expressão de busca, tais como termos relacionados ao sucesso de projetos, tipologia de projetos e métodos 
Fernandes, P. J. A., \& Rabechini, R. Jr. (2021, Ed. Esp. jan./abr.). O gerenciamento de riscos em projetos gerenciados por abordagens ágeis: uma revisão sistemática da literatura

distintos que não estejam relacionados ao Scrum e Extreme Programming. Além disso, avaliar a maturidade das práticas da abordagem ágil pode trazer resultados interessantes, por meio de frameworks publicados para essa finalidade, tal como o Kanban Maturity Model, também conhecido pelo acrônimo KMM. Por fim, focar a análise em artigos cuja unidade de análise não esteja relacionada a projetos de tecnologia despertará interesse dos mais diversos no âmbito dos projetos.

\section{Referências}

Abrahamsson, P. (2002). Agile Software

Development Methods: Review and

Analysis. VTT Technical Research Centre of Finland.

Alharbi, E., \& Qureshi, M. R. (2014). Implementation of Risk Management with SCRUM to Achieve CMMI Requirements. International Journal of Computer Network and Information Security (IJCNIS), 6, 2025. https://doi.org/10.5815/ijcnis.2014.11.03

Bakker, K., Boonstra, A., \& Wortmann, H. (2010). Does risk management contribute to IT project success? A meta-analysis of empirical evidence. International Journal of Project Management, 28(5), 493-503. https://doi.org/10.1016/j.ijproman.2009.07.0 02

Beck, K. (1999). Extreme Programming Explained: Embrace Change (US Ed edition). Addison-Wesley Professional.

Beck, K. (2000). Extreme Programming Explained: Embrace Change (US Ed edition). Addison-Wesley Professional.

Beck, K. (2001). Manifesto for Agile Software Development. https://agilemanifesto.org/

Beck, K. (2004). Extreme Programming Explained: Embrace Change (US Ed edition). Addison-Wesley Professional.
Boehm, B. (1991). Software risk management: Principles and practices. IEEE Software, 8(1), 32-41.

https://doi.org/10.1109/52.62930

Boehm, B. (2000). Requirements that handle IKIWISI, COTS, and rapid change.

Computer, 33(7), 99-102.

https://doi.org/10.1109/2.869384

Boehm, B. (2002). Get ready for agile methods, with care. Computer, 35(1), 6469. https://doi.org/10.1109/2.976920

Boehm, B. (1989). Software risk management. In C. Ghezzi \& J. A. McDermid (Orgs.), ESEC '89 (p. 1-19). Springer Berlin Heidelberg.

Boehm, B., \& Turner, R. (2003). Using risk to balance agile and plan-driven methods. Computer, 36(6), 57-66. https://doi.org/10.1109/MC.2003.1204376

Boehm, B., Turner, R., Booch, G., Cockburn, A., \& Pyster, A. (2003). Balancing Agility and Discipline: A Guide for the Perplexed, Portable Documents (Edição: 1). AddisonWesley Professional.

Boehm, B., Turner, R., Booch, G., Cockburn, A., \& Pyster, A. (2004). Balancing Agility and Discipline: A Guide for the Perplexed, Portable Documents (Edição: 1). AddisonWesley Professional.

Bumbary, K. (2016). Using Velocity, Acceleration, and Jerk to Manage Agile Schedule Risk (p. 80). https://doi.org/10.1109/ICISE.2016.21

Callon, M., \& Courtial, J.-P. (1995). Cienciometría: El estudio cuantitativo de la actividad científica: de la bibliometría a la vigilancia tecnológica (V. Arenas, Trad.). Ediciones Trea, S.L.

Carvalho, M. M. de, \& Rabechini Jr., R. (2014). Impact of risk management on project performance: The importance of soft skills. International Journal of Production Research, 53(2), 321-340. https://doi.org/10.1080/00207543.2014.9194 23 
Chen, H.-M., Kazman, R., \& Haziyev, S. (2016). Strategic Prototyping for Developing Big Data Systems. Ieee Software, 33(2), 36-43.

Chow, T., \& Cao, D.-B. (2008). A survey study of critical success factors in agile software projects. Journal of Systems and Software, 81(6), 961-971. https://doi.org/10.1016/j.jss.2007.08.020

Cockburn, A. (2002). Agile Software Development. Addison-Wesley Professional.

Cockburn, A. (2004). Crystal Clear: A Human-Powered Methodology for Small Teams: A Human-Powered Methodology for Small Teams (1 edition). Addison-Wesley Professional.

Cockburn, A., \& Highsmith, J. (2001). Agile software development, the people factor. Computer, 34(11), 131-133. https://doi.org/10.1109/2.963450

Conforto, E. C., Salum, F., Amaral, D. C., Silva, S. L. da, \& Almeida, L. F. M. de. (2014). Can Agile Project Management Be Adopted by Industries Other than Software Development? Project Management Journal, 45(3), 21-34. https://doi.org/10.1002/pmj.21410

Costa, H., Canto, F. L. do, \& Pinto, A. L. (2020). GOOGLE SCHOLAR METRICS AND NEW THE NEW QUALIS EVALUATION SYSTEM: 17.

Creswell, J. W. (2010). PROJETO DE PESQUISA - METODOS QUALITATIVO, QUANTITATIVO E MISTO: Métodos Qualitativo, Quantitativo e Misto (Edição: $\left.3^{\mathrm{a}}\right)$. Penso.

da Silva, R. E., \& Neto, J. S. (2015). Hiring software development with agile methods in the Brazilian Federal Administration: Key risks and mitigations. Revista Do Servico Publico, 66(1), 97-120.

Dingsøyr, T., Moe, N., Fægri, T., \& Amdahl Seim, E. (2018). Exploring software development at the very large-scale: A revelatory case study and research agenda for agile method adaptation. Empirical Software Engineering, 23, 1-31. https://doi.org/10.1007/s10664-017-9524-2

Doh, J. (2015). From the Editor: Why we need phenomenon-based research in international business. Journal of World Business, 50, 609-611. https://doi.org/10.1016/j.jwb.2015.08.002

Donmez, D., \& Grote, G. (2018). Two sides of the same coin-How agile software development teams approach uncertainty as threats and opportunities. Information and Software Technology, 93, 94-111. https://doi.org/10.1016/j.infsof.2017.08.015

Dybå, T., \& Dingsøyr, T. (2008). Empirical studies of agile software development: A systematic review. Information and Software Technology, 50(9), 833-859. https://doi.org/10.1016/j.infsof.2008.01.006

Ghobadi, S., \& Mathiassen, L. (2017). Risks to Effective Knowledge Sharing in Agile Software Teams: A Model for Assessing and Mitigating Risks. Information Systems Journal, 27(6), 699-731. https://doi.org/10.1111/isj.12117

González-Albo, B., \& Bordons, M. (2011). Articles vs. proceedings papers: Do they differ in research relevance and impact? A case study in the Library and Information Science field. Journal of Informetrics, 5(3), 369-381.

https://doi.org/10.1016/j.joi.2011.01.011

Gusmão, C. M. G. de, \& Moura, H. P. de. (2003). ISO, CMMI and PMBOK Risk Management: A Comparative Analysis. The International Journal of Applied Management and Technology, 1 . https://docplayer.net/9143777-Iso-cmmiand-pmbok-risk-management-acomparative-analysis.html

Hammad, M., Inayat, I., \& Zahid, M. (2019). Risk Management in Agile Software Development: A Survey. https://doi.org/10.1109/FIT47737.2019.0003 9 
Highsmith, J. (1997). Messy, exciting, and anxiety-ridden: Adaptive software development. (Vol. 10).

Highsmith, J. (2002). Agile Software Development Ecosystems (Edição: 1). Addison-Wesley Professional.

Highsmith, J., \& Cockburn, A. (2001). Agile software development: The business of innovation. Computer, 34(9), 120-127. https://doi.org/10.1109/2.947100

Howell, D., Windahl, C., \& Seidel, R. (2010). A project contingency framework based on uncertainty and its consequences.

International Journal of Project

Management, 28(3), 256-264.

https://doi.org/10.1016/j.ijproman.2009.06.0 02

Larman, C., \& Basili, V. R. (2003). Iterative and incremental developments. A brief history. Computer, 36(6), 47-56. https://doi.org/10.1109/MC.2003.1204375

Lee, O.-K. D., \& Baby, D. V. (2013). Managing dynamic risks in global it projects: Agile risk-management using the principles of service-oriented architecture. International Journal of Information Technology \& Decision Making, 12(06), 1121-1150. https://doi.org/10.1142/S021962201340011 7

Lorenzini Erdmann, A., Palucci Marziale, M. H., Gonçalves Pedreira, M. da L., Lana, F. C. F., Freitag Pagliuca, L. M., Itayra Padilha, M., \& Dumêt Fernandes, J. (2009). A avaliação de periódicos científicos qualis e a produção brasileira de artigos da área de enfermagem. Revista Latino-Americana de Enfermagem, 17(3).

http://www.redalyc.org/resumen.oa?id=2814 21908019

Maccari, E. A., \& Nishimura, A. T. (2014). Povoamento dos estratos conceitos 6 e 7 no sistema de avaliação da CAPES pela área de administração, ciências contábeis e turismo nas avaliações trienais 2010 e 2013. Revista Eletrônica de Administração, 20(3), 601624.
Mishra, A., Sinha, K. K., \& Thirumalai, S. (2017). Project Quality: The Achilles Heel of Offshore Technology Projects? Ieee

Transactions on Engineering Management, 64(3), 272-286.

https://doi.org/10.1109/TEM.2017.2662021

Moe, N. B., Dingsøyr, T., \& Dybå, T. (2010). A teamwork model for understanding an agile team: A case study of a Scrum project. Information and Software Technology, 52(5), 480-491. https://doi.org/10.1016/j.infsof.2009.11.004

Montesi, M., \& Owen, J. M. (2008). From conference to journal publication: How conference papers in software engineering are extended for publication in journals. Journal of the American Society for Information Science and Technology, 59(5), 816-829. https://doi.org/10.1002/asi.20805

Mousaei, M., \& Javdani, T. (2018). A New Project Risk Management Model based on Scrum Framework and Prince2 Methodology. International Journal of Advanced Computer Science and Applications, 9(4). https://doi.org/10.14569/IJACSA.2018.0904 61

Nerur, S., Mahapatra, R., \& Mangalaraj, G. (2005). Challenges of Migrating to Agile Methodologies. Commun. ACM, 48(5), 72 78.

https://doi.org/10.1145/1060710.1060712

Odzaly, E. E., Greer, D., \& Stewart, D. (2018). Agile risk management using software agents. Journal of Ambient Intelligence and Humanized Computing, 9(3), 823-841. https://doi.org/10.1007/s12652-017-0488-2

Palmer, S. R., \& Felsing, J. M. (2002). A Practical Guide to Feature-Driven Development (1 edition). Prentice Hall.

Perkusich, M., Gorgonio, K. C., Almeida, H., \& Perkusich, A. (2017). Assisting the continuous improvement of Scrum projects using metrics and Bayesian networks. Journal of Software-Evolution and Process, 
29(6), e1835.

https://doi.org/10.1002/smr.1835

Petticrew, M., \& Roberts, H. (2008).

Systematic Reviews in the Social Sciences: A

Practical Guide (Edição: 1). WileyBlackwell.

PMI. (2013). A Guide to the Project Management Body of Knowledge (Pmbok Guide) - 5th Edition (Edição: Fifth Edition, Fifth edition). Project Management Institute.

PMI. (2017). Project Management Institute. A Guide to the Project Management Body of Knowledge (PMBOK® Guide)-Sixth Edition (1 edition). Project Management Institute.

Poppendieck, M., \& Poppendieck, T. (2003). Lean Software Development: An Agile Toolkit. Addison-Wesley Professional.

Pressman, R. S. (2011). Engenharia de Software. Uma Abordagem Profissional (Edição: $7^{\mathrm{a}}$ ). Mc Graw Hill.

Ramos-Rodríguez, A.-R., \& Ruíz-Navarro, J. (2004). Changes in the Intellectual Structure of Strategic Management Research: A Bibliometric Study of the "Strategic Management Journal", 1980-2000. Strategic Management Journal, 25(10), 981-1004. JSTOR.

Schwaber, K., Beedle, M., \& Martin, R. C. (2002). Agile Software Development with Scrum (Edição: 1). Prentice Hall.

Schwaber, K., Beedle, M., \& Martin, R. C. (2004). Agile Software Development with Scrum (Edição: 1). Prentice Hall.

Senapathi, M., \& Drury-Grogan, M. L. (2017). Refining a model for sustained usage of agile methodologies. Journal of Systems and Software, 132, 298-316. https://doi.org/10.1016/j.jss.2017.07.010

Serrador, P., \& Pinto, J. (2015). Does Agile work? - A quantitative analysis of agile project success. International Journal of Project Management, 33(5), 1040-1051. https://doi.org/10.1016/j.jproman.2015.01.0 06
Shmueli, O., \& Ronen, B. (2017). Excessive software development: Practices and penalties. International Journal of Project Management, 35(1), 13-27. https://doi.org/10.1016/j.ijproman.2016.10.0 02

Shrivastava, \& Rathod, U. (2014). Risks in Distributed Agile Development: A Review. Procedia - Social and Behavioral Sciences, 133, 417-424. https://doi.org/10.1016/j.sbspro.2014.04.208

Shrivastava, \& Rathod, U. (2015).

Categorization of risk factors for distributed agile projects. Information and Software Technology, 58, 373-387. https://doi.org/10.1016/j.infsof.2014.07.007

Shrivastava, \& Rathod, U. (2017). A risk management framework for distributed agile projects. Information and Software Technology, 85, 1-15. https://doi.org/10.1016/j.infsof.2016.12.005

Stapleton, J., \& Constable, P. (1997). DSDM: A framework for business centered development (Edição: 1). Addison-Wesley Professional.

Stoneburner, G., Goguen, A. Y., \& Feringa, A. (2002). SP 800-30. Risk Management Guide for Information Technology Systems.

National Institute of Standards \& Technology.

Sundararajan, S., Bhasi, M., \& Vijayaraghavan, P. K. (2014). Case study on risk management practice in large offshoreoutsourced Agile software projects. IET Software, 8(6), 245-257. https://doi.org/10.1049/iet-sen.2013.0190

Tavares, B. G., da Silva, C. E. S., \& de Souza, A. D. (2019). Practices to Improve Risk Management in Agile Projects. International Journal of Software Engineering and Knowledge Engineering, 29(03), 381-399. https://doi.org/10.1142/S021819401950016 5

Tomanek, M., \& Juricek, J. (2015). Project Risk Management Model Based on PRINCE2 and Scrum Frameworks. 
Fernandes, P. J. A., \& Rabechini, R. Jr. (2021, Ed. Esp. jan./abr.). O gerenciamento de riscos em projetos gerenciados por abordagens ágeis: uma revisão sistemática da literatura

International Journal of Software Engineering and Applications, 6. https://doi.org/10.5121/ijsea.2015.6107

Tranfield, D., Denyer, D., \& Smart, P. (2003). Towards a Methodology for Developing Evidence-Informed Management Knowledge by Means of Systematic Review. British Journal of Management, 14(3), 207-222.

https://doi.org/10.1111/1467-8551.00375
Wanderley, M., Menezes, J., Gusmão, C., \& Lima, F. (2015). Proposal of Risk Management Metrics for Multiple Project Software Development. Procedia Computer Science, 64, 1001-1009.

https://doi.org/10.1016/j.procs.2015.08.619

Zupic, I., \& Čater, T. (2015). Bibliometric Methods in Management and Organization. Organizational Research Methods, 18(3), 429-472.

https://doi.org/10.1177/1094428114562629. 